\title{
On Drag Reduction and Heat Transfer in Turbulent Channel Flow over Circular Dimples: The Shift of the Deepest Point of Dimples
}

\author{
Yong Eng ${ }^{1}$, C.M.J. Tay ${ }^{2}$, B.C. Khoo ${ }^{3}$ \\ ${ }^{1,2,3}$ National University of Singapore \\ Kent Ridge, Singapore 119260 \\ e0023457@u.nus.edu; tsltcm@nus.edu.sg; tslhead@nus.edu.sg
}

\begin{abstract}
In this study, the flow characteristics and enhanced heat transfer performance of circular dimples in a channel flow were numerically analysed and compared with a flat channel. The effect of shifting the deepest point of the dimples in turbulent flow on their drag and heat transfer performance are also discussed. The strength and extent of the induced recirculating flow is suppressed significantly when the deepest point is shifted downstream, enhancing the heat transfer performance of the dimpled wall. At the same time, the flow structure above the dimpled wall is manipulated by the geometry changes. The flow impingement on the dimpled wall increases drag; consequently, the power required to drive the flow is increased. A parametric study is conducted to optimize the shifting of the deepest point to maximize heat transfer performance while minimizing the drag increase.
\end{abstract}

Keywords: Turbulent channel flow, dimple, drag reduction, heat transfer.

\section{Introduction}

Enhancement of heat transfer performance is highly desirable for cooling, with many works focusing on passive methods such as riblets, pin-fins and protrusions [1]. Among these, dimpled surfaces are attractive due to its high heat transfer performance while requiring less additional power to drive the flow. There are many researchers working on heat transfer enhancement using dimpled surfaces. Dimpled surfaces are more power efficient in boosting convective heat transfer compared to other heat transfer enhancing methods [2], [3]. Previous studies suggest even the potential of achieving drag reduction with enhanced heat transfer by using passive circular dimples [3], [4] compared to a plane flat surface. On the other hand, the friction factor of the circular dimpled channel is doubled for some cases done by Rao et al. [5]. The heat transfer enhancement of circular dimple is reported to be about 1.25 times that of the flat channel as Reynolds number increases while the friction factor remains at about 1.55 in the results obtained by Xie et al. [6]. It highlights the fact that the drag and heat transfer performance of circular dimpled surfaces is highly sensitive to the dimpled geometry and flow conditions [7].

Dimples with higher depth to diameter ratios $(d / D)$ have shown increased heat transfer enhancement in turbulent channel flows. However, the drag on the dimpled wall also typically increases with the depth to diameter ratio. The additional drag acting on the dimpled wall requires greater power to maintain the same flow rate within a channel [6]. A region of flow recirculation usually occurring at the upstream portion of the dimple is also not desirable for both drag and heat transfer performance. Recently, teardrop shape dimples have also been studied [8]-[10]. One of the key findings is that the recirculating flow induced by teardrop dimples is weaker than that induced by circular dimples, leading to increased heat transfer performance of the teardrop dimples. The teardrop dimples suppress the recirculating flow region due to a reduced upstream wall slope of the teardrop dimples. This same reduction in upstream wall slope can also be achieved in circular dimples by shifting the deepest point of the circular dimples to boost their heat transfer performance.

To minimize the drag increase, non-axisymmetric circular dimples with different deepest point locations are examined. Experiments show that the greatest drag reduction is observed for such dimples with their deepest point shifted downstream by 0.1 diameter from the dimple center [11]. In the present study, shallow circular dimples with their deepest point shifted in the streamwise direction are considered to achieve minimum drag and maximum heat transfer efficiency. The effect of the dimples is carefully examined in terms of Nusselt number, friction coefficient and flow pattern. 


\section{Numerical Methodology}

The three-dimensional turbulent channel with and without dimples is investigated numerically in the present work. This work focuses on the turbulent flow and heat transfer characteristics above the dimpled wall for dimples with its deepest point at various locations. The turbulent flat channel flow is simulated with flat walls in the present study as the baseline case. For the dimpled cases, the turbulent dimpled channel flow is simulated with one dimpled wall at the bottom with the other opposite wall being flat and without dimples. In this study, the origin of the Cartesian coordinate system is located at the middle of the inlet of the computational domain. The $x, y$ and $z$ axis are the streamwise, wall normal and spanwise directions, respectively. The fluid flows inside a channel with a channel height of $2 H$. The temperature $T$ in this study is considered as a passive scalar [12]. The governing equations of incompressible flow are as follow:

$$
\begin{gathered}
\frac{\partial u_{i}}{\partial x_{i}}=0 \\
\rho\left(\frac{\partial u_{i}}{\partial t}+\frac{\partial u_{i} u_{j}}{\partial x_{j}}\right)=-\frac{\partial p}{\partial x_{i}}+\mu \frac{\partial^{2} u_{i}}{\partial x_{j} x_{j}}+F_{i} \\
\frac{\partial T}{\partial t}+\frac{\partial\left(T u_{i}\right)}{\partial x_{i}}=\alpha \frac{\partial^{2} T}{\partial x_{i} \partial x_{i}}
\end{gathered}
$$

An external force $F_{i}$ is implemented in the $x$ direction to drive the flow through the channel. In this study, it is used to maintain a constant bulk velocity $U_{b}$ in the channel. The turbulent drag reduction characteristics with the dimpled wall were examined at a fixed bulk velocity $U_{b}$. The half channel height $H$ and bulk velocity $U_{b}$ are used for the purpose of non-dimensionalization unless specified. Hence, the Reynolds number is fixed to be the same as the benchmark case for plane channel flow [12]. In this investigation, the Reynolds number $(R e)$ is defined as follows:

$$
R e=\frac{2 H U_{b}}{v} \approx 5600
$$

The bulk velocity is defined for both the dimpled and flat channel as

$$
U_{b}=\frac{\iint u_{x} d A_{x}}{\iint d A_{x}}
$$

where $A_{x}$ is the channel cross-sectional area normal to the $x$ axis. The Nusselt number is defined as

$$
N u=\frac{h D_{h}}{\lambda}
$$

where the local heat transfer coefficient $h$, bulk fluid temperature $T_{r e f}$ and hydraulic diameter are defined as

$$
\begin{gathered}
h=\frac{q}{T-T_{\text {ref }}} \\
T_{\text {ref }}=\frac{\iiint u_{x} T d A_{x} d x}{\iiint u_{x} A_{x} d x} \\
D_{h}=\frac{4 V}{A_{w}}
\end{gathered}
$$

where $V$ is the volume of the computational domain and $A_{w}$ is the total wetted surface area of both the top and bottom walls. The friction factor acting on the wall is expressed as 


$$
C_{f}=\frac{f}{\frac{1}{2} \rho U_{b}{ }^{2}} .
$$

where $f$ is the summation of the skin friction and form drag. The Prandtl number of the working fluid is taken as

$$
\operatorname{Pr}=\frac{v}{\alpha}=0.71
$$

where $v$ and $\alpha$ are the kinematic viscosity and thermal diffusivity, respectively.

Figure 1 shows the computational domain of the dimpled wall channel. Periodic boundary conditions for velocity, pressure and temperature are employed at the streamwise and spanwise directions. No-slip velocity conditions are applied at both the top flat wall and bottom dimpled wall. A constant heat flux is prescribed on the top and bottom walls. The working fluid in this study is dry air. The fluid's thermal physical properties are assumed to be constant. Direct Numerical Simulation is conducted using the open source code OpenFOAM [13]. The finite volume method is applied with 2nd order accuracy in space and time. Backward time scheme is applied to time-stepping algorithm. The residual convergence criteria adopted at every time step is $1 \times 10^{-6}$ for both velocity and pressure. The sampling time is doubled until it shows less than $0.01 \%$ difference in drag. The time averaged and turbulence statistics presented in this study are collected until statistically stable. The computational work for this article was done on resources at the National Supercomputing Computer, Singapore (https://www.nscc.sg).

In the simulation, the domain is discretized in a structured mesh with streamwise grid size $\Delta x^{+}=7.8$ and spanwise grid size $\Delta z^{+}=5.9$. In order to resolve the near-wall flow in the Direct Numerical Simulation, at least 3 grids points should lie within the viscous sublayer along the wall normal direction [14]. The thickness of the first mesh layer in the wall normal direction in the present study is $\Delta y_{w}^{+}=0.04$; and the thickness of the cell at the middle of the channel $\Delta y_{c}^{+}=4.4$. The flat channel flow was executed in a $12.6 H \times 2 H \times 4.2 H$ domain with $\Delta x^{+}=14.4$, spanwise grid size $\Delta z^{+}=5.9, \Delta y_{w}^{+}=0.04$ and $\Delta y_{c}^{+}=4.4$. The non-dimensional time step $\Delta t^{+}$is 0.002 .

\subsection{Configuration of New Dimple Shape}

Figure 1 shows the cross section along the diameter of the axisymmetric circular dimple and the computational domain of the dimpled channel. The dimples are placed in a staggered arrangement on the lower wall. The depth to diameter ratio $d / D$ is fixed at 0.05 for all the shallow dimples in this study. To examine the heat transfer capability, these dimples are skewed in the streamwise direction by a simple shear deformation performed on the axisymmetric dimple. The deepest point is originally located at the center of the axisymmetric dimple. It is shifted either in the upstream or downstream directions in the skewing operation. In order to quantify the magnitude of the shift, $\Delta x / D$ is defined as the ratio of the displacement of the deepest point from the center of the dimple $\Delta x$ to the dimple diameter $D$. The parameters of the skewed dimple considered in the present study are presented in Table 1 . The circular axisymmetric dimple is skewed in the streamwise direction by simple shear deformation:

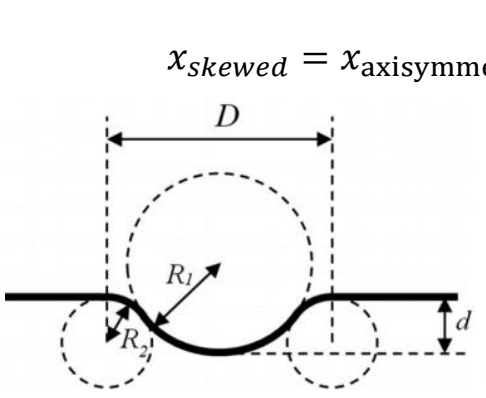

(a)

Fig. 1 (a) Cross sectional view of dimple geometry with $\Delta x / D=0$, (b) dimple simulation domain (right). 
Table 1 The parameters for different dimple configurations. Case $3(\Delta x / D=0)$ represents the axisymmetric dimple.

\begin{tabular}{c|c|c|c|c|c|c}
\hline Case & $D / H$ & $d / H$ & $d / D$ & $R 1 / R 2$ & $R 1 / D$ & $\Delta x / D$ \\
\hline Flat & 0 & - & - & - & - & - \\
1 & 5 & 0.25 & 0.05 & - & - & -0.2 \\
2 & 5 & 0.25 & 0.05 & - & - & -0.1 \\
3 & 5 & 0.25 & 0.05 & 0.5 & 0.84 & 0 \\
4 & 5 & 0.25 & 0.05 & - & - & +0.1 \\
5 & 5 & 0.25 & 0.05 & - & - & +0.2 \\
\hline
\end{tabular}

\section{Result and Discussion}

The time averaged velocity profile, Reynolds stresses and temperature profile of the plane channel without dimples are compared with published results to validate the numerical methods and results [12], [15]. Comparisons are also made of $\mathrm{Nu}$ and $C_{f}$ with previous studies. To ensure that the flow is fully developed, the data is collected after 50 to 70 flow through times in the streamwise direction. The flat channel result shows good agreement with previous works. The Nusselt number is in good agreement with that obtained from Kays and Crawford [16]. The non-dimensional temperature profile also matches well with other published DNS work [12]. This approach is next extended to the dimpled channel cases by maintaining the bulk velocity $U_{b}$.

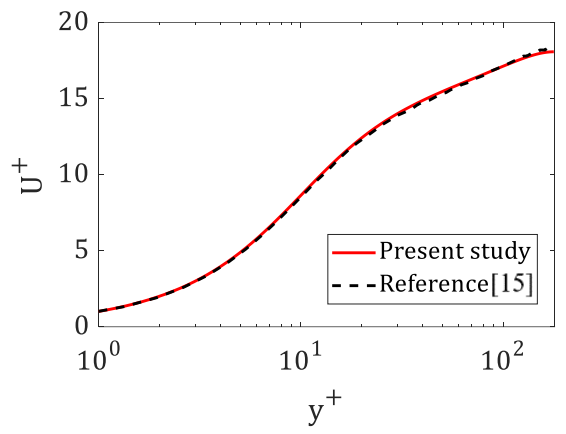

(a)

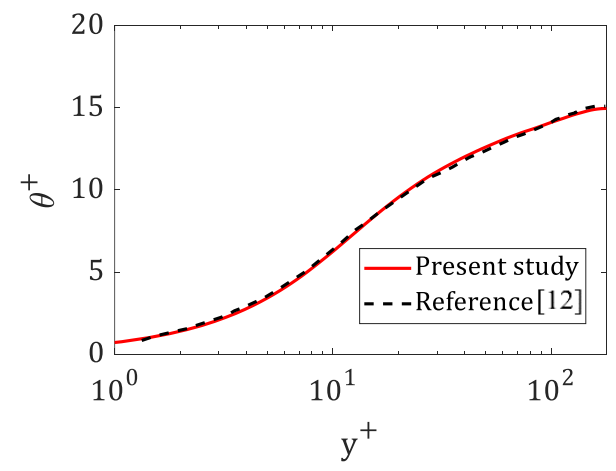

(c)

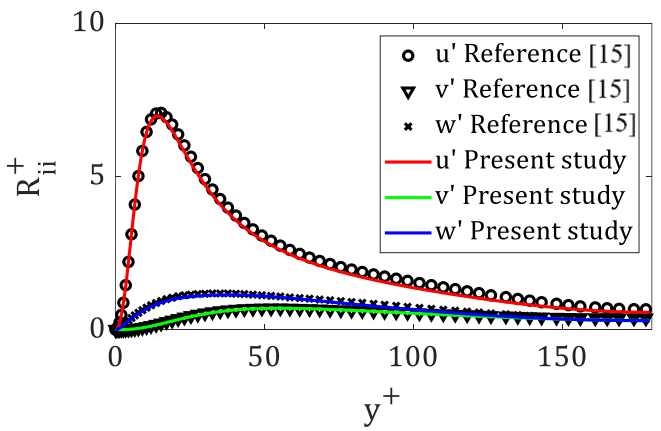

(b)

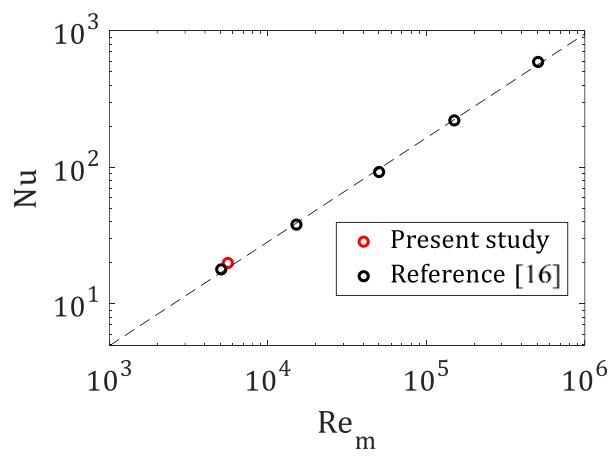

(d)

Fig. 2 (a) Velocity profiles (b) Reynolds stresses (c) Temperature profiles (d) Nusselt number for flat channel case compared with previous works [12] [15] [16]. 


\subsection{Global Thermo-Aerodynamic Performance}

In this section, the objective is to elucidate the effect of movement of the deepest point of shallow circular dimples on both the heat transfer efficiency and drag performance. The impact of shifting the deepest location in the dimple is presented and discussed in this section. To explore the heat transfer efficiency and drag performance in a consistent manner, thermoaerodynamic parameters are introduced by comparing with the flat channel case at the same Reynolds number. These parameters are presented in terms of the friction factor ratio $C_{f} / C_{f 0}$ and the Nusselt number ratio $N u / N u_{0}$. The subscript 0 represents the flat channel case. These parameters are both spatial and time-averaged quantities which represent the overall performance of the dimpled wall. Figure 3 shows that the friction factor ratio and Nusselt number ratio are greater than unity across all the dimpled channels. This suggests that all the dimpled channels have better heat transfer efficiency than the flat channel. The Nusselt number ratio remains at about $+7 \%$ between $\Delta x / D=0$ and +0.2 . As the deepest location is being shifted upstream, the heat transfer efficiency improves. The greatest improvement is with $\Delta x / D=-0.2$ at $+14.12 \%$. The significant differences of heat transfer capability for different deepest location are hence presented.

At the same time, the drag acting on the dimpled channel is $+13.26 \%$ greater than the flat channel when the deepest location is shifted to $\Delta x / D=-0.2$. For $\Delta x / D=-0.2$, the dimpled wall slope at the upstream portion is the steepest among all the cases examined. As the deepest location is moved downstream, the additional drag acting on the wall reaches its minimum of $+3.76 \%$ at $\Delta x / D=+0.1$. This observation coincides with the reduction of slope at the upstream locality of the dimple. However, the downstream slope is increased to maintain the circular geometry. As the downstream slope is increased further when $\Delta x / D=+0.2$, the friction factor ratio $C_{f} / C_{f 0}$ due to the dimples starts to increase again. The result for the friction factor ratio $C_{f} / C_{f 0}$ is plotted in Figure 3(b) together with previous experimental results [11] for comparison. The result for the axisymmetric dimple (Case 3) geometry shows excellent agreement with the experimental results when extrapolated to the Reynolds number of the present numerical study, while the trend as $C_{f} / C_{f 0}$ varies with $\Delta x / D$ generally agrees with the experimental results [11] for all the dimple geometries considered.

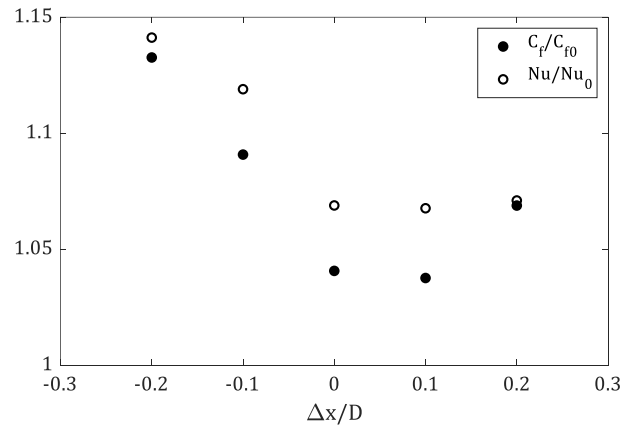

(a)

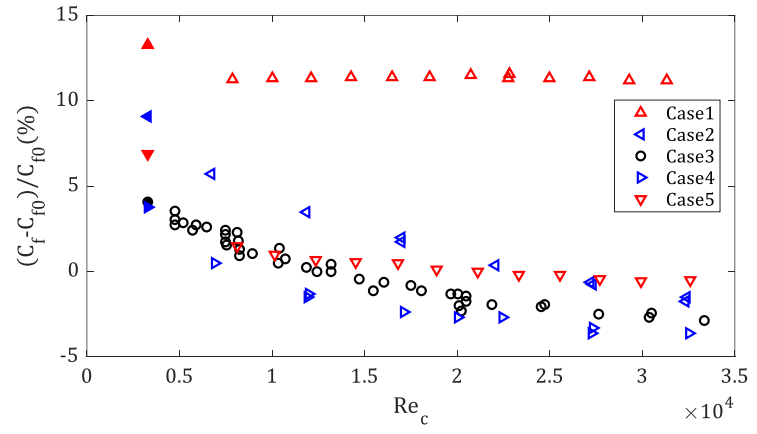

(b)

Fig. 3 (a)Effect of deepest point on friction factor ratio and Nusselt number ratio (b) Drag measurement for all five cases. Solid markers: DNS result; Hollow markers: Experimental work [11].

To further examine the flow, the friction factor ratio $C_{f} / C_{f 0}$ has been decomposed into skin friction $C_{F}$ and form drag $C_{P}$ contributions respectively and normalized by the friction coefficient of the flat channel, $C_{f 0}$. The result is presented in Figure 4. It can be seen clearly that the form drag contribution is strongly related to the location of the deepest point $\Delta x / D$. The additional form drag's magnitude is about $30 \%$ of the drag of the flat channel. The trend of the form drag variation with $\Delta x / D$ is similar to the trend of the friction factor ratio which indicates that the form drag contribution significantly influences the overall drag performance. On the other hand, the skin friction remains about $90 \%$ of that of the flat plane channel for all the dimple cases considered. It shows that all the dimpled cases studied are having less skin friction compared to the flat channel despite having a greater wetted area. 


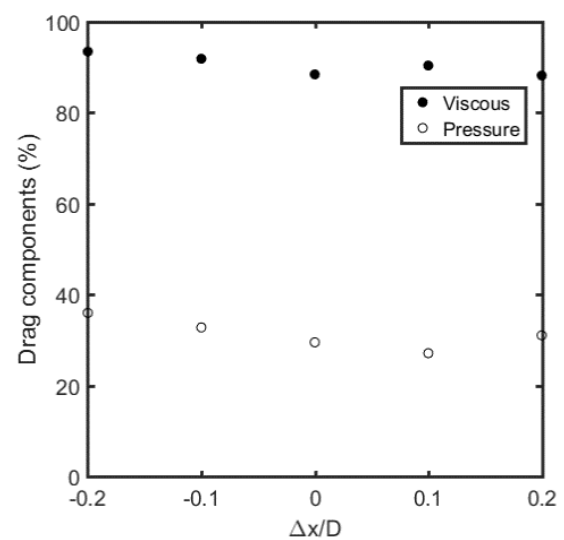

Figure 4: Form drag and skin friction contribution on dimpled wall.

\subsection{Mean Characteristics of Drag and Heat Transfer}

Further examination of the form drag and skin friction acting on the dimpled wall reveals the relationship between the slope of the dimpled wall and friction factor ratio. Figure 5 shows the form drag and skin friction distribution over the dimple surfaces. The black solid lines in the figures represent the edges of the circular dimples, and the white dot indicates the position of the deepest point of the dimple. The flow direction is in the $x$-direction which is from left to right. At negative values of $\Delta x / D$, the region of highest form drag, labelled region I in Figure 5(a) is concentrated within the upstream region of the dimple depression. A lower form drag peak occurs near the dimple center, labelled region II in Figure 5(a), and directly downstream of the peak form drag concentration. Near the downstream edge of the dimple, a minimum form drag exists, labelled III in Figure 5(a). As the deepest point is shifted downstream and $\Delta x / D$ increases, the region of concentrated form drag (I) is weakened and becomes distributed over a larger region spatially. At the same time, both the magnitudes of the second form drag peak (II) and form drag minimum (III) are enhanced significantly and move downstream. As $\Delta x / D$ increases to +0.2 , the upstream from drag concentration (I) has weakened significantly, and the maximum form drag now occurs near the downstream dimple edge (II). This is immediately followed by the form drag minimum (III) directly downstream of it. These significant changes in the peak form drag and form drag minima occurring within the dimple reveals the important role the wall slope plays in the form drag distribution within the dimple. The upstream form drag region (I) dominates the overall form drag performance until the deepest point is shifted far downstream. The steeper the upstream slope, the stronger the upstream form drag region (I).

The skin friction contour on the dimpled wall is presented in Figure 5(b). The blue area indicates the region of flow separation. The skewed dimple with $\Delta x / D=-0.2$ shows the largest magnitude of negative skin friction, indicating the strongest separated flow. As the deepest point moves downstream, the flow separation region shrinks significantly. This separation feature shrinks completely and becomes absent in the case $\Delta x / D=+0.2$. The smooth gentle entry into the dimple depression at the upstream edge when $\Delta x / D=+0.2$ prevents the formation of flow separation. Table 3 shows that the size of the flow separation in the dimple follows the same trend as the form drag until the secondary form drag region (region II and III) dominates the resultant drag. 


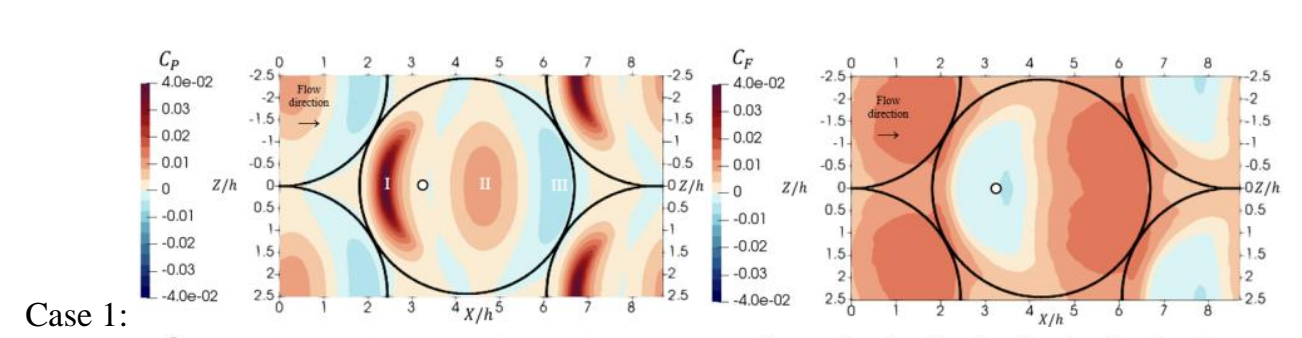

Case 1:

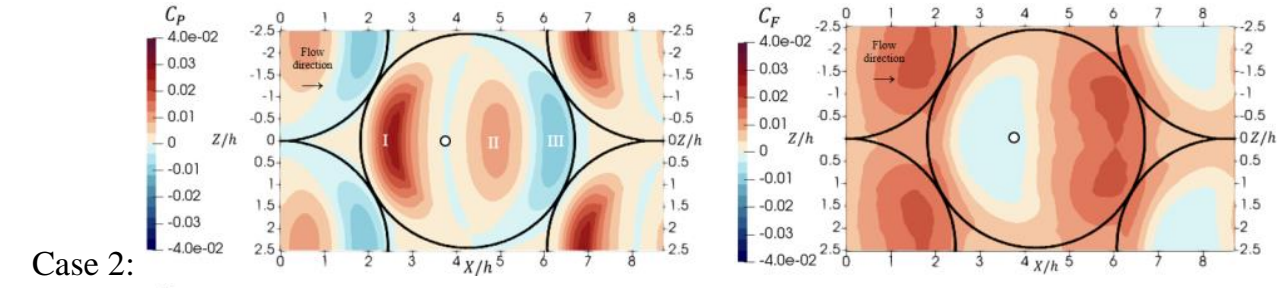

Case 2:
$C_{P}$
\[ \begin{array}{c}4.00-02 \\ -0.03 \\ -0.02 \\ -0.01 \\ -0 \\ -0.01 \\ -0.02 \\ -0.03 \\ -4.00-02\end{array} \]
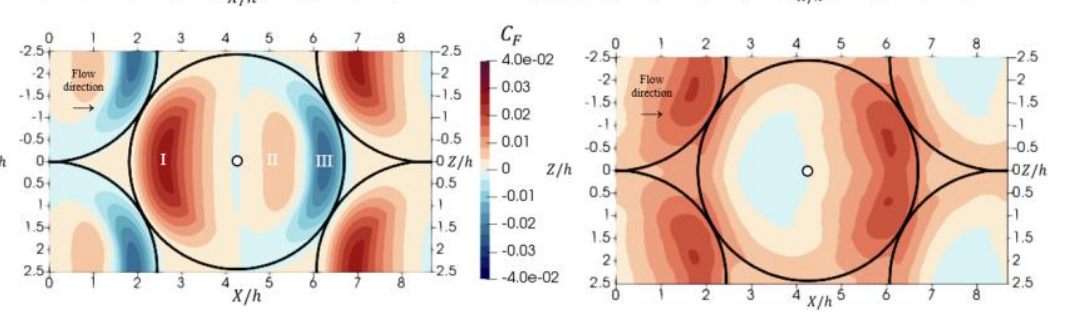

Case 3:
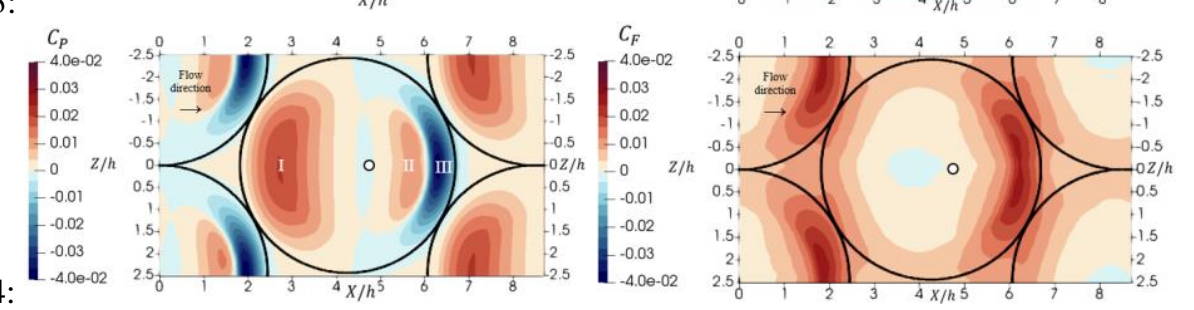

Case 4:

Case 5:

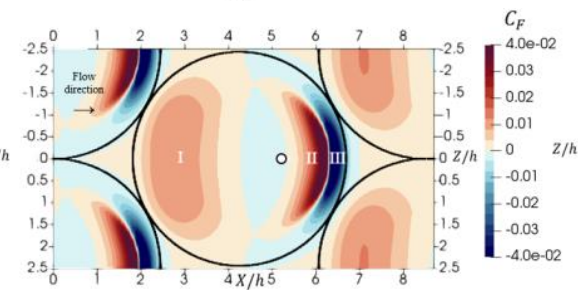

(a)

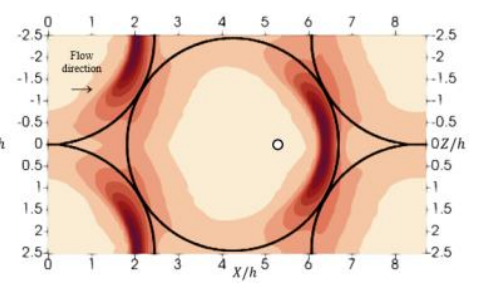

(b)

Fig. 5 (a) Form drag (b) skin friction contour on dimpled wall.

White dot indicates the deepest point. Flow is from left to right.

The Nusselt number distribution on the dimpled wall is presented in Figure 6(a). The heat transfer efficiency is boosted significantly at the downstream side of the dimples throughout all the cases. This enhanced region reaches its peak at the intersections where the dimples of adjacent rows meet as the location of the deepest point shifts downstream. On the other hand, the upstream region shows poorer heat transfer efficiency. This region of lower heat transfer efficiency coincides with the region of flow separation shown in Figure 5(b). There are noticeable changes in the flow within the dimple as the deepest point is shifted in the streamwise direction from $-0.2 D$ to $0.2 D$. Figure $6(\mathrm{~b})$ shows the streamlines above the dimpled wall and explains the reason behind the reduced heat transfer at the upstream portion of the dimples. As the flow enters the dimple, it separates if the upstream wall slope is too steep, resulting in a recirculating region in the upstream portion of the dimple. This flow recirculating region traps fluid mass within this region, reducing movement of the warm fluid away from this region and correspondingly reduces heat transfer. Among all the cases, Case 1 has the strongest flow recirculation at the upstream edge. This flow recirculation region shrinks as $\Delta x / D$ increases and the wall slope decreases. Along with this 
reduction in the size of the recirculating region, the strength of the flow recirculation also becomes weaker as the wall slope reduces. A significant reduction in the size of the recirculation region is observed when $\Delta x / D$ increases from zero, the symmetric case to $+0.1 D$. When $\Delta x / D=+0.2$, the flow recirculation region disappears completely, and no flow separation is observed at the upstream portion of the dimple. This shows that reducing the upstream wall slope is an effective means to weaken or even eliminate the recirculating flow occurring at the upstream portion of the dimple.
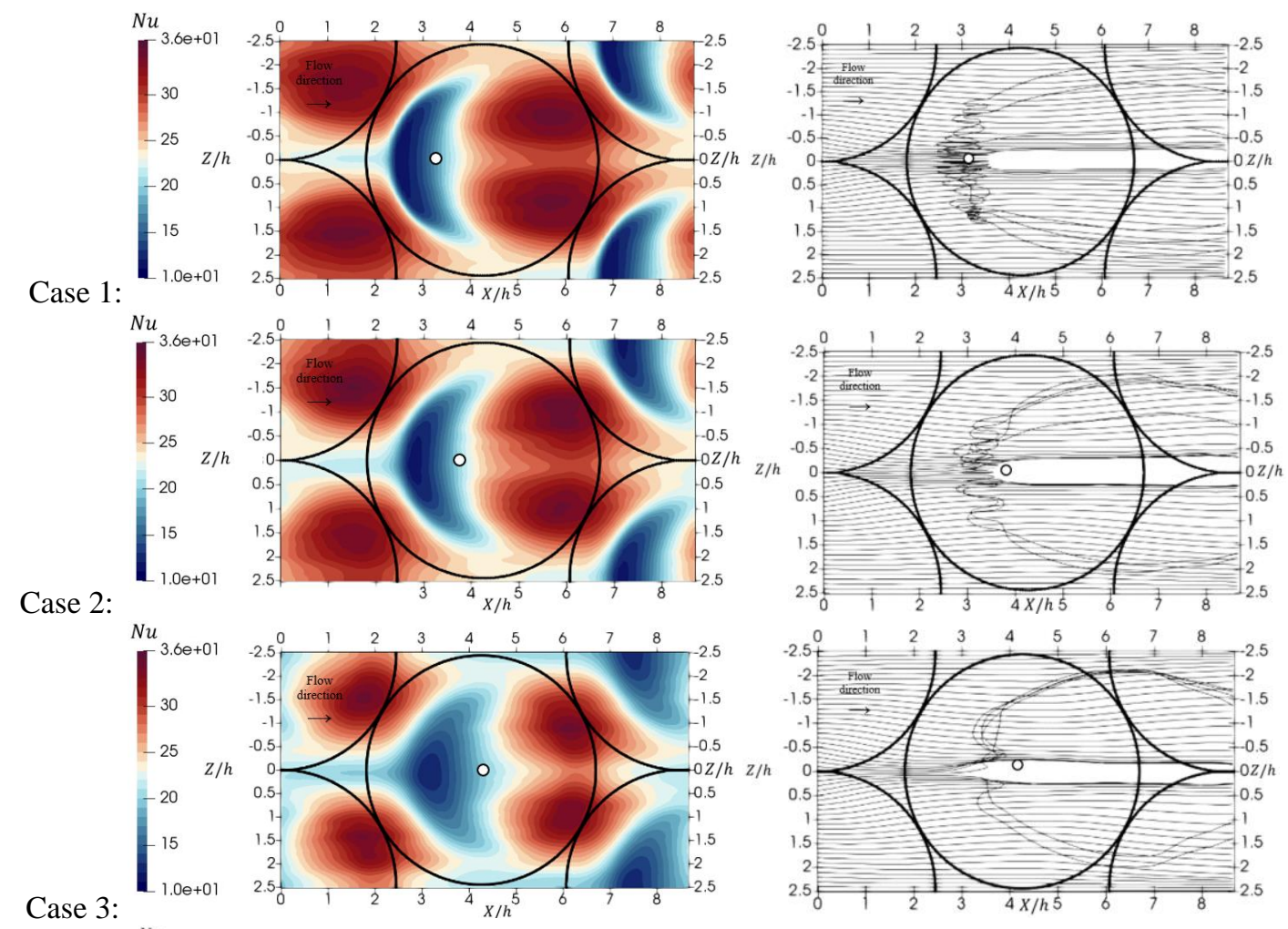

$\coprod_{-30}^{N u}{ }_{-25}^{3.60+01}$
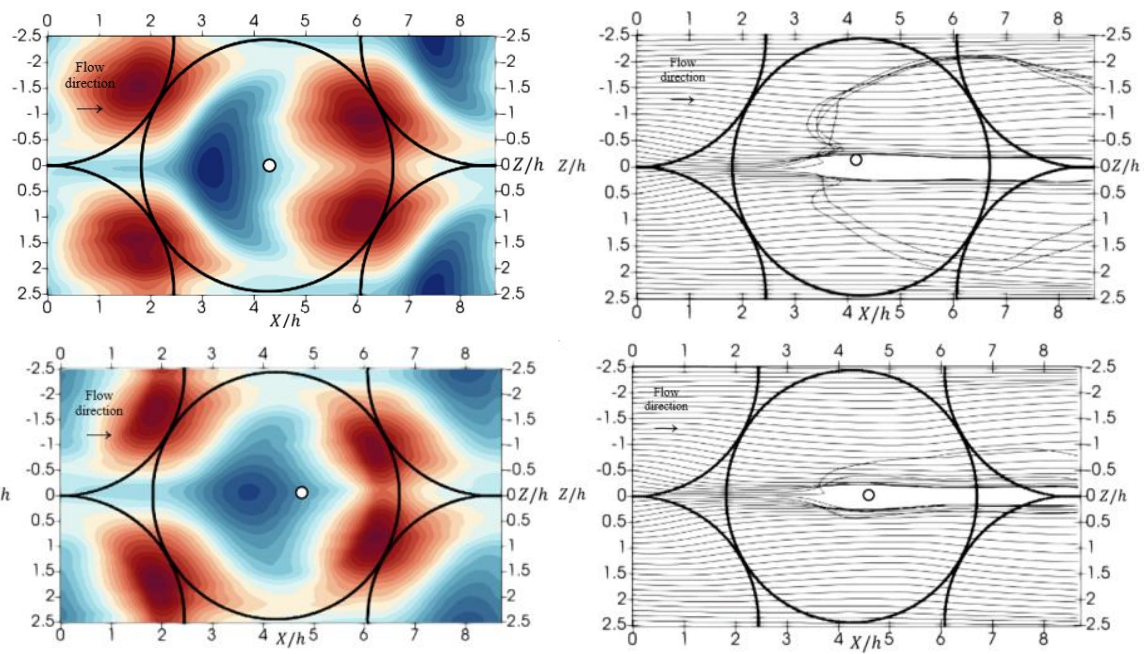

Case 4:

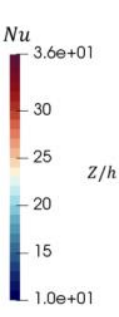

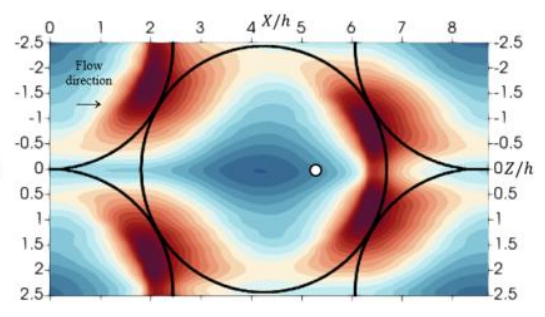

(a)
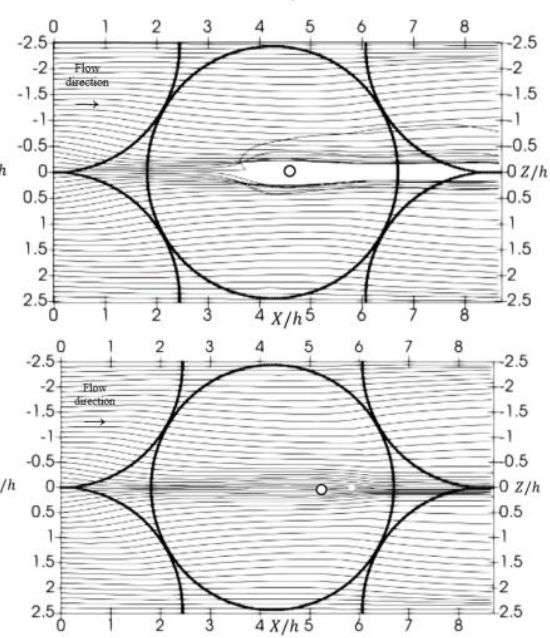

(b)

Fig. 6 (a) Nusselt number (b) streamlines on dimpled wall.

White dot indicates the deepest point. Flow is from left to right. 


\section{Conclusion}

The shallow dimple with varying deepest point in the streamwise direction is examined in terms of its heat transfer efficiency and drag at Reynolds number 5600 and Prandtl number of 0.71 in the present study. The objective is to elucidate elucidate the effect of movement of the deepest point of shallow circular dimples on both the heat transfer efficiency and and drag performance. In the present study, DNS is used to investigate the effect on the flow when the deepest point is shifted shifted either in the upstream and downstream directions through a skewing operation.

Drag acting on the shallow dimpled channel is strongly related to the location of the deepest point $\Delta x / D$. The primary form drag region dominates the overall form drag performance until the deepest point is shifted too far downstream. The steeper the upstream slope, the stronger the flow separation that forms at the upstream dimple edge. The formation of separation can be prevented by skewing the dimple geometry towards the downstream direction. However, the flow impingement phenomenon observed at the downstream dimple wall then becomes stronger, increasing the secondary form drag region. By taking both primary and secondary form drag regions into account, the resultant drag reaches its minimum when the dimple is skewed so that $\Delta x / D=+0.1$.

\section{Acknowledgements}

The computational work for this article was done using resources from the National Supercomputing Computer, Singapore (https://www.nscc.sg).

\section{References}

[1] G. Xie, B. Sundén and W. Zhang, "Comparisons of Pins/Dimples/Protrusions Cooling Concepts for a Turbine Blade Tip-Wall at High Reynolds Numbers," Journal of Heat Transfer, vol. 133, (6), pp. 061902, 2011.

[2] N. K. Burgess and P. M. Ligrani, "Effects of Dimple Depth on Channel Nusselt Numbers and Friction Factors," Journal of Heat Transfer, vol. 127, (8), pp. 839-847, 2005.

[3] Y. Chen, Y. T. Chew and B. C. Khoo, "Enhancement of heat transfer in turbulent channel flow over dimpled surface," International Journal of Heat and Mass Transfer, vol. 55, (25-26), pp. 8100-8121, 2012.

[4] C. M. J. Tay, B. C. Khoo and Y. T. Chew, "Mechanics of drag reduction by shallow dimples in channel flow," Physics of Fluids, vol. 27, (3), pp. 35109, 2015.

[5] Y. Rao, B. Li and Y. Feng, "Heat transfer of turbulent flow over surfaces with spherical dimples and teardrop dimples," Experimental Thermal and Fluid Science, vol. 61, pp. 201-209, 2015.

[6] S. Xie, Z. Liang, L. Zhang, Y. Wang, H. Ding, and J. Zhang, "Numerical investigation on heat transfer performance and flow characteristics in enhanced tube with dimples and protrusions," International Journal of Heat and Mass Transfer, vol. 122, pp. 602-613, 2018.

[7] A. I. Leontiev, N. A. Kiselev, Yu. A. Vinogradov, M. M. Strongin, A. G. Zditovets, and S. A. Burtsev, "Experimental investigation of heat transfer and drag on surfaces coated with dimples of different shape," International Journal of Thermal Sciences, vol. 118, pp. 152-167, 2017.

[8] Q. Jing, Y. Xie and D. Zhang, "Thermal-hydraulic performance and entropy generation of supercritical carbon dioxide in heat exchanger channels with teardrop dimple/protrusion," International Journal of Heat and Mass Transfer, vol. 135, pp. 1082-1096, 2019.

[9] A. Perwez and R. Kumar, "Heat transfer performance investigation of the spherical dimple heat sink and inclined teardrop dimple heat sink," Numerical Heat Transfer. Part A, Applications, vol. 76, (2), pp. 73-86, 2019.

[10] S. Xie, Z. Liang, J. Zhang, L. Zhang, Y. Wang, and H. Ding, "Numerical investigation on flow and heat transfer in dimpled tube with teardrop dimples," International Journal of Heat and Mass Transfer, vol. 131, pp. 713-723, 2019.

[11] J. Tay and T. T. Lim, "Drag Reduction with Non-Axisymmetric Dimples," in 35th AIAA Applied Aerodynamics Conference, Denver, Colorado, Jun. 2017, doi: 10.2514/6.2017-3569.

[12] J. Kim and P. Moin, "Transport of passive scalars in a turbulent channel flow." in Turbulent Shear Flows 6, pp. 85-96. Springer, Berlin, Heidelberg, 1989. 
[13] H. G. Weller, G. Tabor, H. Jasak, and C. Fureby, "A tensorial approach to computational continuum mechanics using object-oriented techniques," Computers in physics, 12(6), pp.620-631, 1998.

[14] J. G. M. Eggels et al, "Fully developed turbulent pipe flow: a comparison between direct numerical simulation and experiment," Journal of Fluid Mechanics, vol. 268, pp. 175-210, 1994.

[15] J. Kim, P. Moin and R. Moser, "Turbulence statistics in fully developed channel flow at low Reynolds number," Journal of Fluid Mechanics, vol. 177, pp. 133-166, 1987.

[16] W. M. Kays, M. E. Crawford and B. Weigand, Convective Heat and Mass Transfer. (4th ed.) New York: McGraw-Hill Higher Education, 2005. 